Der Deutsche Kartographie Kongress wird in seiner 65. Auflage nun zum ersten Mal unter diesem Namen gemeinsam mit der Intergeo in Berlin vom 26.-28. September stattfinden. Der Titel der Kongressmesse wurde langfristig festgelegt und mit „Wissen und Handeln für die Erde" beschreibt er sehr gut, wofür die Geo-Fachverbände und auch die DGfK stehen und welchen gesellschaftlichen Nutzen wir bieten. Die jährlich fortgeschriebenen und mit aktuellen Trends abgeglichenen Themengebiete der Intergeo sind in diesem Jahr Geospatial 4.0, Digital Construction, Open Government und Smart Cities. Damit werden Intergeo und DKK inzwischen deutschlandweit und auch international beworben, wodurch eine große Beteiligung auch in diesem Jahr erwartet wird. Eine ideale Gelegenheit also, Lösungen und Leistungsfähigkeit von Kartographie und Geomatik einer großen Fachöffentlichkeit vorzustellen.

Die Programmverantwortlichen unter der Leitung von Vizepräsident Jochen Schiewe haben den Aufruf zur Einreichung von Vorschlägen herausgegeben und dabei insbesondere den Begriff "Smart" in den Mittelpunkt gestellt sowie die Frage aufgeworfen, welche Beiträge die Kartographie und Geomatik leisten kann, um das Leben für Menschen zufriedenstellender, effizienter und nachhaltiger zu gestalten. Die Bedeutung von "Smart" auch in diesem Zusammenhang kann durchaus mit intelligent oder clever umschrieben werden. Es stellt sich also die Frage, wie Kartographie und Geomatik zur Beantwortung der aktuellen gesellschaftlichen Fragestellungen in den herausgehobenen Bereichen Energie, Mobilität und Stadt sowie weiteren Themen beitragen können. Hier können und sollen die Vorträge - und auch die in der KN erscheinenden Beiträge - des 65. Deutschen Kartographie Kongresses zeigen, dass Kartographie und Geomatik die gesamte Breite der
Darstellung und Kommunikation raum-zeitlicher Informationen abdecken, einschließlich der Bereitstellung von Diensten jederzeit und an jedem Ort.

Auch auf die Ausschreibung zum Ravenstein-Förderpreis 2017 sei an dieser Stelle noch einmal ausdrücklich hingewiesen. Die gute bis sehr gute Beteiligung der Auszubildenden an diesem für die Nachwuchsförderung der DGfK und auch für die berufliche Zukunft der Absolventinnen und Absolventen wichtigen Preis lässt die Frage aufkommen, warum die Zahl der Bewerbungen aus den Universitäten und Hochschulen in den letzten Jahren nur gering war. Die Teilnahmebedingungen des Wettbewerbs betonen die Bedeutung des wissenschaftlichen Aspekts der Arbeiten und auch die Offenheit für die Nachbardisziplinen von Kartographie und Geomatik. Daher sind neben den Ausbilderinnen und Ausbildern insbesondere die Lehrenden an Universitäten und Hochschulen noch einmal aufgerufen, die Studierenden auf den RavensteinFörderpreis hinzuweisen.

Manfred Weisensee, Hude Präsident der DGfK

Fortbildungsaktivitäten der Sektion Rhein-Ruhr

Sektion startete

Workshop-Reihe zum

Einsatz von Open-Source-

Technologien in der Kartographie

Um DGfK-Mitgliedern Fortbildungen zu aktuellen Entwicklungen im praktischen Einsatz von OpenSource-Software in der Kartographie/Geomatik anzubieten, veranstaltet die Sektion RheinRuhr in CIP-Inseln des Geographischen Instituts an der Ruhr-Universität Bochum (RUB) drei Tagesworkshops (jeweils an Samstagen) zu folgenden Themen:

Webmapping mit leaflet.js und GeoJSON (29.4.2017, 10-15 Uhr, Dozent: Dr. Dennis Edler)
Einführung in die Geodatenanalyse mit QGIS (20.5.2017, 10-15 Uhr, Dozent: Gregor Bein, M. Sc.)

Themakartographie mit Inkscape (17.6.2017, 10-15 Uhr, Dozent: Dr. Dennis Edler).

Interessierte Mitglieder anderer Sektionen sind herzlich eingeladen, mit der Sektionsleitung Kontakt aufzunehmen (dennis. edler@rub.de). Zudem ist geplant, das Fortbildungsangebot für die Zukunft zu erweitern. Ideen für weitere Workshops gern unter Beteiligung anderer Dozenten aus der Praxis - sind jederzeit herzlich wilkommen!

Dennis Edler, Bochum
Sektion Südbaden

- Aus der Arbeit

2015/2016

Dieser Bericht der Sektion Südbaden schließt an den in KN 2/2015 auf Seite $117 \mathrm{ff}$. veröffentlichten Beitrag an

\section{Veranstaltungen 2015}

Bei der ersten Veranstaltung des Jahres (22. Januar 2015) präsentierte Dipl.-Geogr. Markus Müller (Abt. Geoinformation und Kartografie, Stadtmessungsamt Stuttgart) das Geo-Portal der Stadt Stuttgart. Man erfuhr unter anderem, dass der Zweck dieses Geo-Portals nicht nur die Bürgerinformation ist, sondern dass auch innerhalb der Stadtverwaltung die Geodaten bekannt ge- macht werden sollen. Daraus ergeben sich als Anwender in Zahlen: 40 Fachämter - 40 Fachverfahren - 300 Datenebenen und 2.000 Nutzer. Das GeoPortal macht u..a. auch eine Zeitreise möglich mit Luftbildern aus den Jahren 2006 - 2009 - 2011 - 2013. Des Weiteren wurde das 3D-Stadtmodell erläutert und auf das Ziel „mobile Nutzung auf Smartphones" hingewiesen. Abschließend gab es für alle Anwesenden der Veranstaltung einen kleinen Neujahrsempfang.

Am 16. April folgte die jährliche Mitgliederversammlung der Sektion. Neben den üblichen Punkten, wie den Berichten der Vorsitzenden, der Sektionsfinanzverwalterin und der Kassenprüfer sowie der Präsentation des Haushaltsplanes, und der Entlastung des Vorstandes, ist folgender Beschluss erwähnenswert: Das 60-jährige Bestehen soll in großem Rahmen in Form eines Kolloquiums gefeiert werden (vgl. KN 1/2017, S. 44). Wegen fehlender bezahlbarer Räumlichkeiten wurde auf eine Kartenausstellung verzichtet.

Im Verlauf der Veranstaltungsreihe gab es in Kooperation mit dem Alemannischen Institut Freiburg einer Serie von Vorträgen zum Thema „Freiburg mit offenen Karten“, die auf das Jahr der Karte 2015/2016 (IMY) hinweisen und es bereichern sollten.

Die Auftaktveranstaltung dazu am 21. Mai bestritt kein geringe-

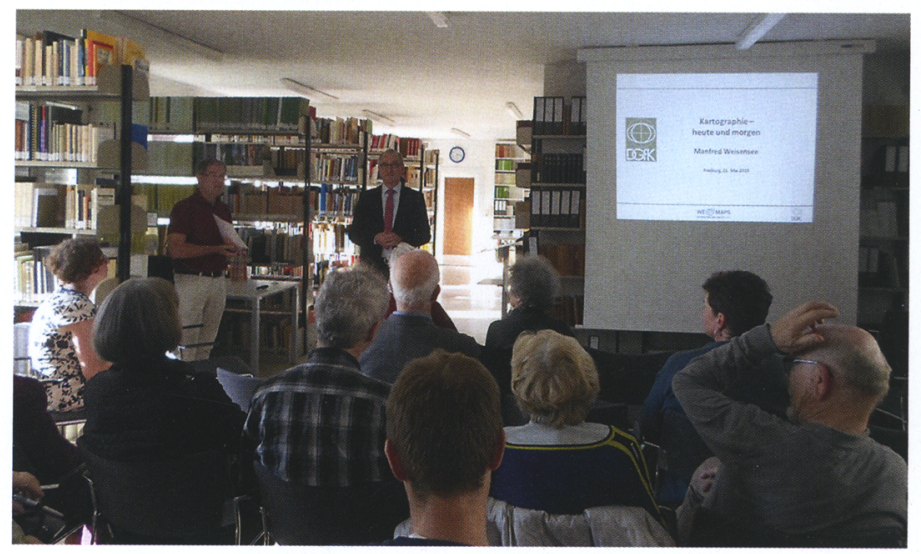

Abb. 1: Der Präsident der DGfK bei seinem Vortrag (Foto: B. Schmücking) 